\title{
Two-Photon Microscopy of Stroma ex vivo for the Express Diagnostics of Breast Cancer
}

DOI: $10.17691 / \mathrm{stm} 2019.11 .3 .12$

Received December 21, 2018

E.A. Sergeeva, PhD, Senior Researcher, Laboratory of Biophotonics ${ }^{1}$;

M.Yu. Kirillin, PhD, Senior Researcher, Laboratory of Biophotonics ${ }^{1}$;

V.V. Dudenkova, PhD, Researcher, Institute of Experimental Oncology and Biomedical Technologies2;

M.V. Pavlov, PhD Student, Department of Oncology, Radiotherapy and Radiology2;

N.Yu. Orlinskaya, MD, DSc, Professor, Principal Researcher of the Group of Pathological Anatomy and Tissue Preservation, University Clinic;

N.M. Shakhova, MD, DSc, Leading Researcher, Laboratory of Biophotonics ${ }^{1}$

${ }^{1}$ Federal Research Center Institute of Applied Physics, Russian Academy of Sciences, 46 Ulyanova St., Nizhny Novgorod, 603950, Russia;

2Privolzhsky Research Medical University, 10/1 Minin and Pozharsky Square, Nizhny Novgorod, 603005, Russia

The goal of the study is to develop the approach for the express analysis of ex vivo biopsy specimens of breast tissue based on twophoton microscopy (TPM).

Materials and Methods. We studied breast tissue samples obtained by trephine biopsy procedure. TPM images of breast stroma obtained from the ex vivo biopsy specimens were compared with TPM images of the unstained deparaffinized 10- $\mu$ m thick histological sections of breast tissue. The study included samples of patients with fibroadenoma (2 cases), in situ carcinoma ( 1 case), and invasive carcinoma of non-specific type I-II grade (4 cases). In the frames of numerical processing of TPM images, collagen disorganization factor maps based on spatial Fourier analysis were constructed.

Results. We have demonstrated the feasibility of the TPM technique in detecting stroma remodeling in breast biopsy specimens in the development of benign and malignant changes. Typical images of biopsy specimens and histological sections were obtained for normal breast tissue, fibroadenoma and various types of carcinomas. Distinction between typical images of benign and malignant tumors was demonstrated. Changes in collagen fibers structure and the presence/absence of elastin fibers in the considered pathological cases were revealed. Differences in TPM images of biopsy specimens and histological sections are shown, which may originate from the distortion of fiber shape in the course of histological sample preparation. The proposed algorithm for quantitative TPM image processing allows objectifying the results of imaging, which is an essential step towards automated express diagnostics.

Conclusion. Two-photon microscopy has high potential as the express biopsy technique for the primary diagnosis of breast tissue, as well as for intraoperative use.

Key words: two-photon microscopy; second harmonic generation; two-photon excited autofluorescence; breast; stroma; fibroadenoma; carcinoma.

\section{Introduction}

Breast cancer is currently the most common type of cancer in women worldwide, and demographic trends indicate a continuous increase in incidence rate. According to forecasts, only in the European Union by 2020 about 394,000 new cases of breast cancer will be registered yearly together with 100,000 deaths among patients with breast cancer [1]. Over the past decades, breast cancer treatment approaches have undergone significant progress, and current trend is the implementation of organ-preserving operations [2-4] requiring a reliable intraoperative assessment of surgical margins. The problem is, malignant tumors are typically heterogeneous, and commonly used frozen-section rapid histological analysis has a number of limitations related with both tumor heterogeneity and the coldinduced deformation of the sample during preparation, which affect the diagnostics quality. In this regard, the development of methods allowing for fast morphological analysis of large tissue volumes without additional processing is of high importance.

Recently, optical express biopsy approaches for cancer diagnostics have been developed [5-7]. These approaches are based on the principles of optical multispectral visualization of bioissue cellular structure and allow for retrieving information on morphological type of the tumor, the presence of malignant cells in the surgical margin during organ-preserving operations, the presence of specific molecular markers [8], including

Corresponding author: Ekaterina A. Sergeeva, e-mail: sea_nnov@yahoo.com 
those for breast cancer [9]. Rapid optical biopsy methods allow for imaging both in vivo and ex vivo postoperative samples (so-called "fresh tissue samples").

Two-photon microscopy (TPM) is one of the promising technologies for optical biopsy of biological tissue native samples [10], which was used for the analysis of tumors of various localizations [11-15]. The TPM approach is based on the principle of laser scanning microscopy [16], in which an infrared femtosecond pulsed laser is used as a source, and the image is formed by simultaneously registering second harmonic generation (SHG) signals from collagen and two-photon-excited autofluorescence (TPAF) from elastin and cell cytoplasm components in the visible spectral range. The TPM approach allows for imaging of the structure of biological tissues with micron spatial resolution and does not require additional staining, which satisfies the requirements for intraoperative use.

The goal of this work is to develop the approach to fast ex vivo analysis of biopsy samples of breast tissue based on TPM imaging technique. In the paper, the feasibility of TPM for tracking changes in breast stroma structure with benign and malignant alterations was studied using trephine biopsy samples obtained in the course of tumor diagnostics. Typical TPM images of normal breast tissue, fibroadenoma, and various carcinoma types were obtained, and qualitative differences between typical images of breast benign and malignant neoplasms were revealed. The changes in collagen fibers organization and the presence or absence of elastin fibers were demonstrated in the considered breast pathologies. After imaging of biopsy specimens and routine histological examination, TPM technique was applied to image the structure of breast stroma in unstained deparaffinized histological sections of the same specimens. As a result of the comparison, distinctive features of certain pathologies were revealed in TPM images of biopsy samples and histological sections. Numerical processing of TPM images of collagen fibers is proposed, which can be further employed to develop an algorithm for automated recognition of pathologies in breast tumors diagnostics.

\section{Materials and Methods}

Two-photon microscopy. The study involved biopsy samples of breast tissue obtained from 7 patients, diagnosed with fibroadenoma (2 cases), carcinoma in situ (1 case) and invasive carcinoma of a nonspecific type of I-II grade (4 cases) according to the results of histological examination. Biopsy material in the form of breast tissue column was obtained during a routine trephine biopsy procedure performed under ultrasound control. Within $60 \mathrm{~min}$ after the biopsy, the unstained samples were imaged with a Carl Zeiss LSM 510 META laser scanning microscopy system (Germany) equipped with an inverted microscope. A Mai Tai HP pulsed femtosecond laser (Spectra Physics, USA) with a pulse repetition rate of $80 \mathrm{MHz}$ and pulse duration of 100 fs was used for fluorescence excitation. Samples were placed on a 170- $\mu$ m-thick coverslip. Imaging was performed in the reflective mode using an oil-immersion objective EC Plan-Neofluar 40×/NA=1.3 (Carl Zeiss, Germany). Images with the size of $1024 \times 1024$ pixels were constructed by scanning a sharply focused laser beam over the corresponding field of view equal to $318 \times 318 \mu \mathrm{m}$. The average laser radiation power in the sample plane was less than $2 \mathrm{~mW}$. TPM imaging was performed upon excitation at a wavelength of $800 \mathrm{~nm}$, SHG signal from collagen was recorded in the spectral range of 385-415 nm, and TPAF signal from elastin and cells was recorded in the range of $460-600 \mathrm{~nm}$. The resulting image is an overlay of SHG and TPAF images where SHG and TPAF signals were encoded in green and red, respectively. After TPM imaging, the samples were subjected to a standard histological examination.

Histological examination. Biopsy samples fixed in a solution of neutral $10 \%$ formalin were subjected to standard histological examination using an Excelsior ES (Thermo Scientific, USA) setup. After preparation, paraffin blocks were fabricated using the HistoStar embedding workstation (Thermo Scientific, USA). Serial 4-6 $\mu$ m-thick sections were sliced with a Microm HM 325 microtome (Thermo Scientific, USA). The sections were stained with hematoxylin and eosin using a Gemini AS automated slide stainer (Thermo Scientific, USA), followed by a routine histological examination using a Leica 2500 microscope (Leica Microsystems, Germany). Slices with a thickness of $10 \mu \mathrm{m}$ obtained from the same blocks were deparaffinized and examined using the TPM technique without additional staining.

Numerical processing. To quantify the degree of collagen fibers organization, spatial Fourier analysis of TPM images was employed. Principles of such an analysis were previously proposed in [17] and later applied to assess the structure of artery walls [18, 19] and age-related skin changes [20, 21]. In this study, SHG images of breast biopsy samples acquired by registering collagen nonlinear optical response were analyzed. Two-dimensional Fourier spectrum of an image with the structured fibers is usually represented by a bell-like shape in the low spatial frequency region. The cross-section of the 2D spectrum at a certain empirically chosen level can be approximated by an ellipse. We define the ratio of the short to long axis of this ellipse as the collagen disorganization factor (CDF) ranging from 0 to 1 . The tendency of CDF to 1 means the equality of the ellipse axes (i.e. ellipse turns to circle), which corresponds to the absence of a distinguished direction in the fiber orientation and their disorganization. CDF values close to 0 mean a significant difference in the axes length, which indicates the presence of a particular direction in fiber orientation and their ordering (Figure 1). The program for calculating CDF from SHG images is implemented in the MATLAB environment.

Since a particular SHG image may contain regions 
with different collagen organization patterns, the original images were divided into 4 equal squares $512 \times 512$ pixels in size to increase the information content. For each of the smaller squares CDF was calculated and a corresponding collagen disorganization map was constructed for the full image. The color palette of the map is chosen in such a way that the red areas correspond to the areas with the highest collagen disorganization, and the blue areas correspond to the areas of ordered collagen in the original image.

\section{Results}

Figures 2-5 demonstrate TPM images of breast biopsy samples (hereinafter referred to as "ex vivo TPM images"), TPM images of the unstained $10-\mu \mathrm{m}$ histology sections (hereinafter referred to as "TPM images of sections") and the corresponding images of stained histological sections. It should be emphasized that the fields of view in ex vivo TPM images and TPM images of sections from the corresponding sample are not absolutely co-localized, we focus on a qualitative comparison of the typical structures revealed within a certain biopsy
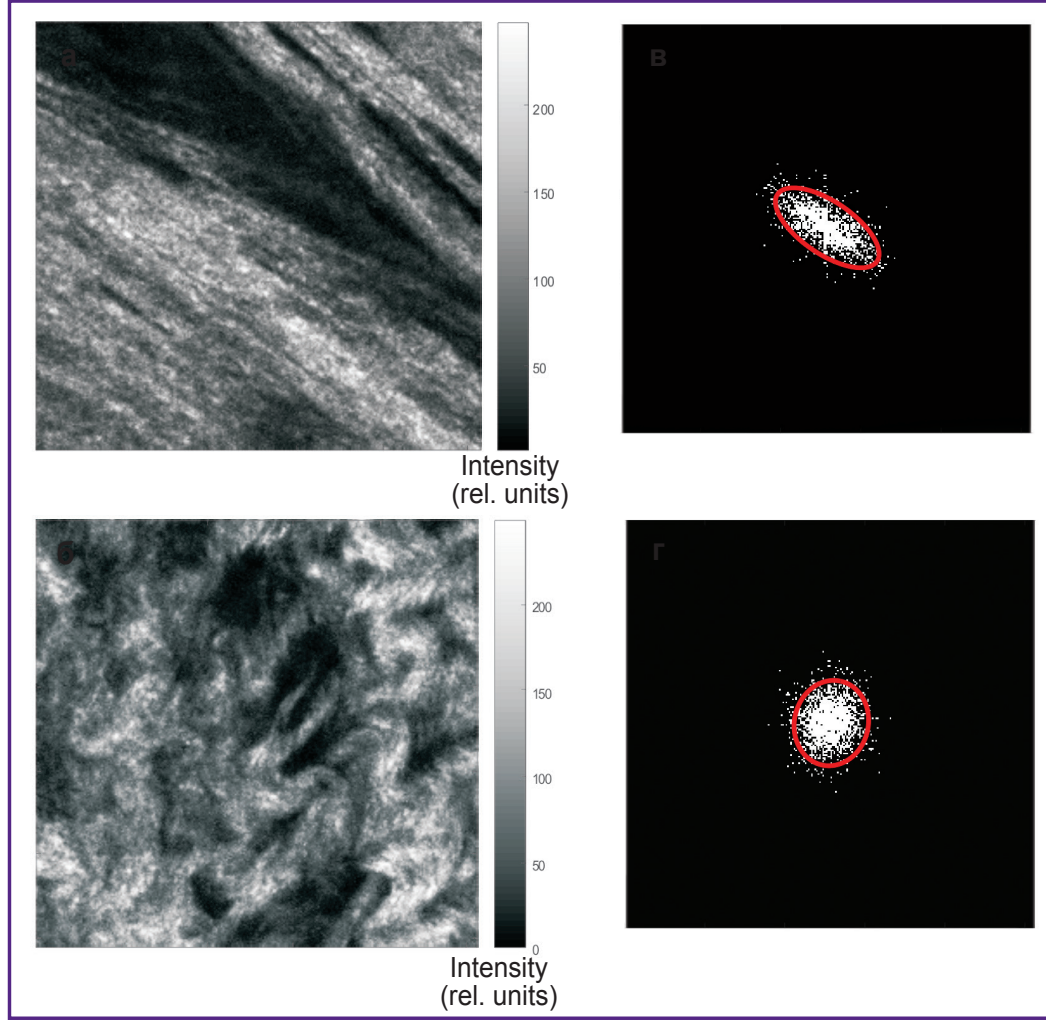

Figure 1. Examples of collagen disorganization factor calculation SHG-images with high (a) and low (b) organization of collagen fibers (image size: $318 \times 318 \mu \mathrm{m})$ and the corresponding $2 \mathrm{D}$ Fourier transform images $(\mathrm{c}),(\mathrm{d})$ (size: $256 \times 265$ pixels) with the ellipse fit. $C D F=0.44$ (a), (c); $C D F=0.85$ (b), (d)

\section{sample.}

Normal tissue. Figure 2 shows images of normal breast fibrous tissues excised from the side region of a sample with fibroadenoma. Histological examination confirms that the visualized area corresponds to the normal stroma of the breast (Figure 2 (c)). Ex vivo TPM image demonstrates that the stroma of normal tissue is loose, collagen fibers are thin (up to $5 \mu \mathrm{m}$ thick), long, straight, mostly ordered and are characterized by weak SHG signal. Elastin fibers and epithelial cells are not visualized in TPAF image. In TPM image of an unpainted section of normal tissue, collagen fibers exhibit a wavy structure. This mismatch between the ex vivo TPM images and TPM images of slices presumably originates from the sample processing. Elastin fibers in TPM image of slices are not visualized as well.

Fibroadenoma. In the case of fibroadenoma (see Figure 3), breast stroma becomes dense, and ex vivo TPM images demonstrate bundles of collagen fibers of various thickness, both long and fragmented, with a wavy fiber direction. Fibrous tissue covers almost the
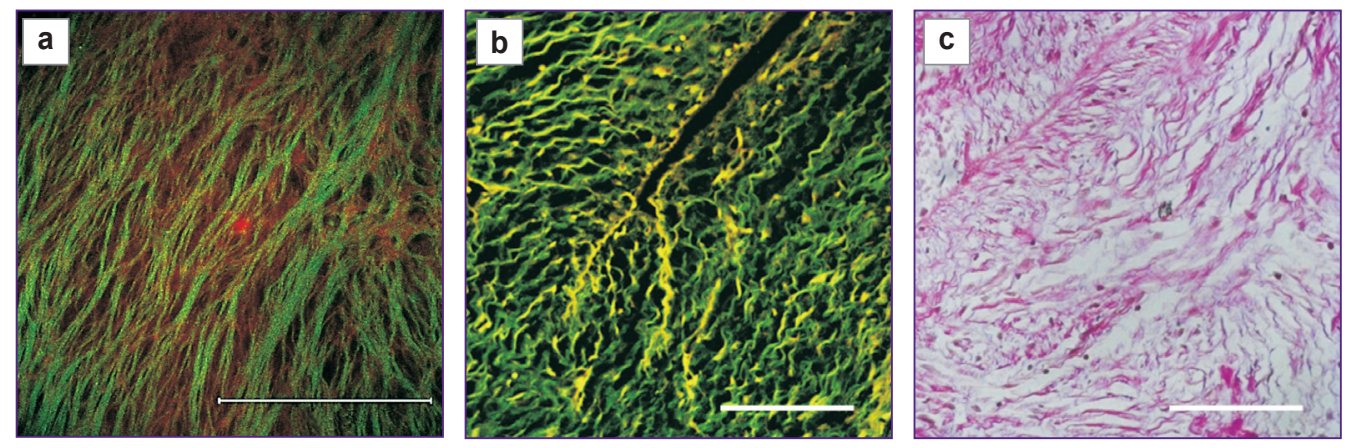

Figure 2. TPM images of ex vivo sample (a), unstained section (b), and histology image (c) of normal breast tissue

Bar equals $100 \mu \mathrm{m}$. SHG signal from collagen is encoded with green, TPAF signal from elastin and cells is encoded with red 
entire field of view with no particular direction of fibers, although there are small clusters of co-directed bundles. The thickness of individual fibers is of the order of few microns, while the thickness of the bundles reaches tens of microns. Similar to normal tissue, elastin fibers and epithelial cells are not visualized in TPAF images. TPM images of sections also show dense stroma consisting of randomly oriented bundles of collagen fibers. Some images show cross-sections of mammary gland ducts with epithelial cells covering their surface visualized in TPAF images, as well as cross-sections of blood vessels walls visualized by SHG signal.

In situ carcinoma. In general, ex vivo TPM images of breast stroma in this case (see Figure 4) are similar to those for fibroadenoma, however, in some areas, among the dense wavy collagen fibers, thin elastin fibers are visualized as twisted tangles or in the shape of bound filaments, while epithelial cells are not visualized.
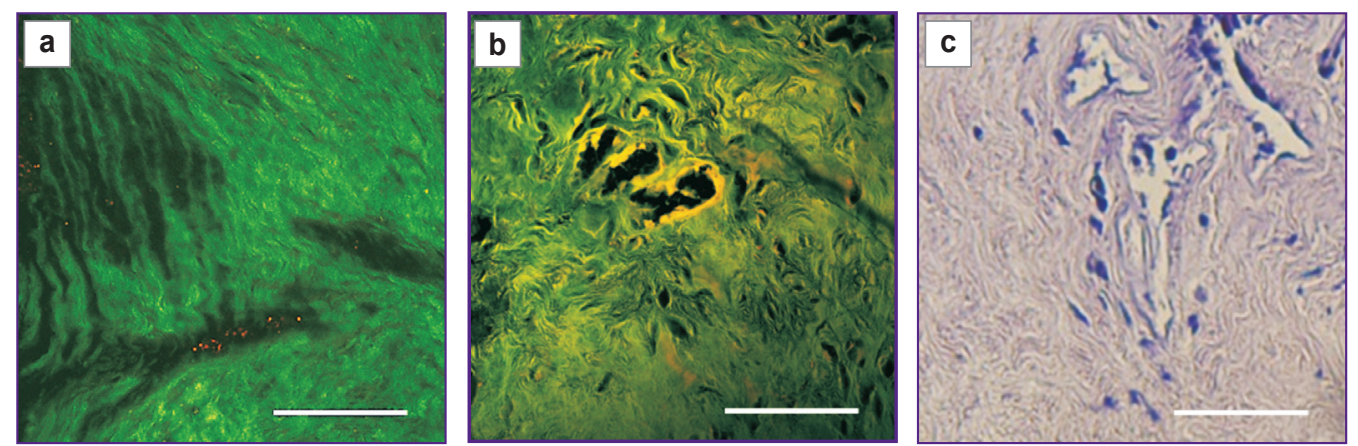

Figure 3. TPM image of ex vivo sample (a), unstained section (b), and histology image (c) of fibroadenoma

Bar equals $100 \mu \mathrm{m}$. SHG signal from collagen is encoded with green, TPAF signal from elastin and cells is encoded with red
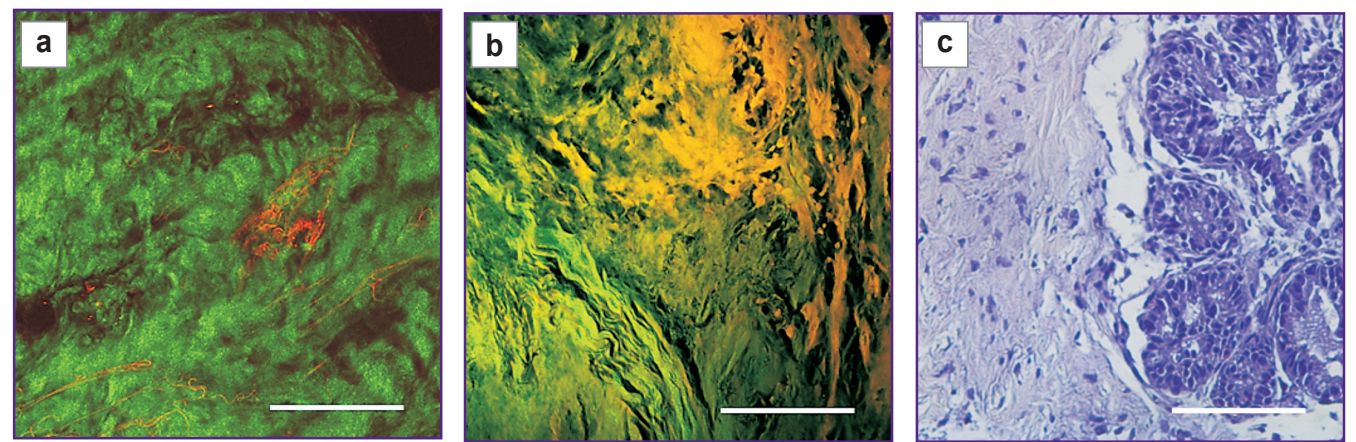

Figure 4. TPM image of ex vivo sample (a), unstained section (b), and histology image (c) of in situ carcinoma

Bar equals $100 \mu \mathrm{m}$. SHG signal from collagen is encoded with green, TPAF signal from elastin and cells is encoded with red
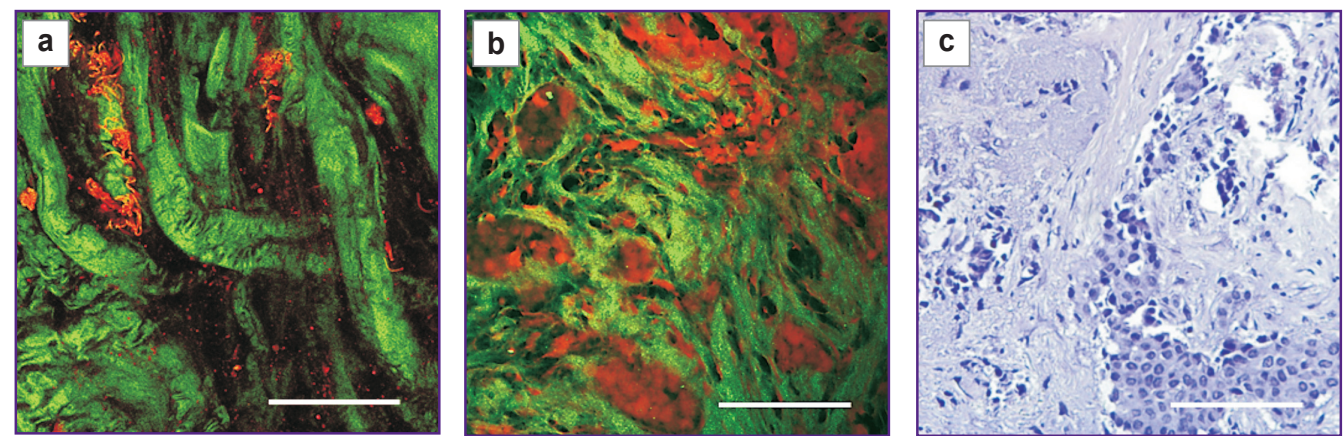

Figure 5. TPM image of ex vivo sample (a), unstained section (b), and histology image (c) of invasive carcinoma

Bar equals $100 \mu \mathrm{m}$. SHG signal from collagen is encoded with green, TPAF signal from elastin and cells is encoded with red 
At the same time, TPM image of unstained sections reveals a region with a large localized agglomeration of cells. This section neighbors the area of dense long and wavy collagen fibers, among which individual thin elastin fibers are visible. Comparison with histology demonstrates that the revealed region may belong to the tumor in situ.

Invasive carcinoma of a nonspecific type of I-II grade. In ex vivo TPM images elongated thick collagen bundles are visualized with the thickness of up to $20 \mu \mathrm{m}$ (see Figure 5). Local density of collagen fibers forming the bundles is rather high, however, the bundles themselves are quite fragmented. Due to the beginning of the hyalinosis process, it is impossible to distinguish individual collagen fibers in TPM images of bundles. Ex vivo TPM images are characterized by pronounced heterogeneity of stromal structures: both long and thick bundles of collagen fibers and thinner wavy structures with a higher density are present in the field of view. Bundles of elastin fibers up to $5 \mu \mathrm{m}$ thick are also observed, epithelial cells are not visualized. Similar to the ex vivo TPM images, TPM images of sections show substantially altered, thickened collagen fibers in stroma, but additionally, numerous evenly spaced cells not localized within the ducts, which correlates well with histological images (Figure 5 (c)). At the same time, elastin fibers are rarely found in TPM images of sections.

Numerical processing. The degree of collagen fibers organization in biopsy samples stroma was evaluated using the described numerical algorithm for all the considered cases. CDF distribution maps are shown in Figure 6 together with the corresponding SHG images. As expected, in normal stroma the degree of coorientation of the fibers is the largest, and the CDF value within the field of view does not exceed 0.4. For fibroadenoma, the degree of collagen disorganization is more pronounced: there are both areas with ordered collagen for which CDF does not exceed 0.4 , and areas with dense packing
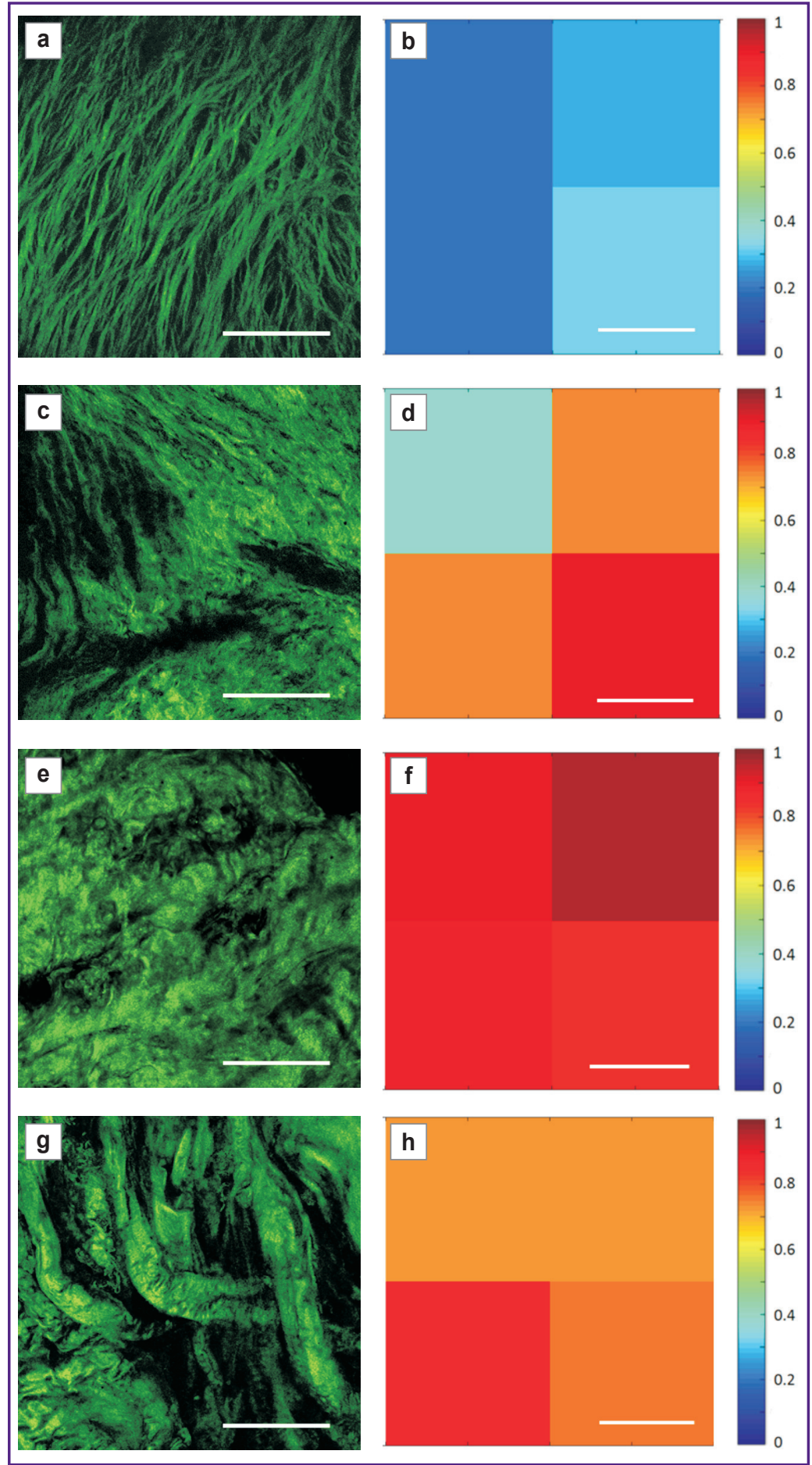

Figure 6. SHG images of collagen in normal breast tissue (a), fibroadenoma (c), carcinoma in situ (e), and invasive carcinoma (g) and corresponding disorganization factor maps (b), (d), (f), (h)

Bar equals $100 \mu \mathrm{m}$ of disoriented fibers, for which CDF values are in the range of $0.7-0.9$. For carcinoma, CDF values are in a wide range $(C D F=0.6-1)$, which indicates high degree of heterogeneity of collagen organization: lower CDF values correspond to the areas with thick straight collagen bundles, while large CDF values correspond to areas with wavy structures. In general,

numerical processing allows us to differentiate between healthy and altered breast tissue stroma, however, for more detailed recognition of various pathologies it is necessary to combine CDF distribution maps with the information about collagen fibers thickness and the presence of elastin fibers. 


\section{Discussion}

Implementation of TPM technique for the diagnostics of breast tumors is limited by difficulties in interpreting the obtained data, in particular, in establishing the correspondence between TPM images and the images obtained by traditional methods of morphological analysis of biological tissues. Currently, microscopy of hematoxylin and eosin stained histological sections with contrasted cell nuclei is the "gold standard" tool in oncology diagnostics. By now, the main diagnostic component of biological tissue is nuclei of epithelial cells, namely, their localization, amount and type. In contrast to histological examination, TPM approach provides information typically on the structure of breast tissue stroma, mostly on collagen and elastin organization and to a lesser extent, on the characteristics of epithelial cells nuclei [22]. Thus, direct comparison of histology images with TPM images is complicated and the value of the information obtained by TPM technique is questionable within the framework of the traditional histological paradigm. However, according to modern studies, extracellular matrix of breast tissue is very sensitive to the changes in the structure of epithelial cells parenchyma that forms lobules and ducts. As the result of epithelial cells mutation and their growth inside stroma, the parenchymal-stromal relation also changes leading to alterations in the structure of collagen fibers and elastin [23-25].

As it was shown in tumor models of laboratory animals, collagen remodeling occurs under the impact of tumor secreted oncoproteins and growth factors, while the fibers network formed in this way provides tumor cells with favorable conditions for invasion and metastasis [26-31]. Benign changes in the breast tissue (in particular, fibroadenomatosis) are associated with excessive proliferation of the connective tissue component of stroma, which also affects the state of collagen and elastin [24]. Thus, TPM has diagnostic value in the detection of breast lesions, which is confirmed by a number of papers [15, 32-35]. High correlation of sequential changes in the organization of collagen fibers with the degree of invasion of breast tumors was observed in laboratory animals, and typical signs of remodeled collagen structure were classified as tumor-associated collagen signatures [30]. However, vast majority of papers on TPM employment for the diagnosis of human breast cancer basing on the changes of stroma structure were performed at fixed sections [32-36], and in some cases, at histological hematoxylin and eosin stained sections prepared for a clinical morphological study [34, 36]. As a result of sample processing, stroma organization in the studied section can be significantly disrupted in comparison with the native biological tissue, while TPM study of stained sections can lead to artifacts and misinterpretation due to pronounced fluorescence of eosin. Besides, typically, TPM analysis of breast stroma state is mainly limited by the analysis of collagen structure, while elastin, another fibrous component of extracellular matrix, is usually ignored (possibly due to a disintegration of its structure in the course of sample processing or due to spectral overlap of elastin autofluorescence with eosin fluorescence). At the same time, it is known from the literature [37-39] that elastosis accompanies certain malignant changes in breast, and, therefore, TPM visualization of elastin fibers organization seems a promising approach.

In this paper it was demonstrated that TPM images obtained from ex vivo biopsy samples of breast tissue differ significantly from those obtained from unstained sections. In particular, the structure of collagen fibers in normal tissue is different: in ex vivo images, the fibers are straight, while in the images of sections, they are wavy. In the papers devoted to imaging breast tissue stroma with various pathologies using unstained sections, the wave-like shape of thin collagen fibers is associated with normal biological tissue. In our opinion, a distortion of the shape of the fibers occurs during the processing of the samples. Also, we did not observe cells in ex vivo samples, which makes it difficult to compare TPM data with the results of standard histological examination. On the other hand, in the ex vivo TPM images of tissues with malignant changes, elastin fibers are clearly visualized, which is extremely rare in TPM images of sections, and perhaps it is the reason why elastin is not properly considered in the literature. We suggest that the presence or absence of elastin fibers in TPM images may serve a marker of malignant changes, and, therefore, this issue requires a more thorough analysis. In addition, further classification of TPAF images also requires consideration of such factors as the age and receptor status of a patient.

\section{Conclusion}

Two-photon microscopy technique used for ex vivo imaging of breast tissue samples in norm and pathology demonstrated the distinctive features of stroma under various conditions, which were not detected in the images of unstained tissue sections and by standard histological examination. The obtained results can be used for intraoperative diagnosis of breast tumors by the TPM technique and for future development of multiphoton systems with an external scanning head for in vivo imaging. The proposed algorithm for quantitative image processing based on the Fourier analysis enables to objectify the results of visualization, which is a significant step towards automated express diagnostics.

Acknowledgements. The authors are grateful to S.V. Gamayunov for useful discussions.

Funding. The work is supported by Russian Foundation for Basic Research (RFBR), \#16-02-00974.

Conflict of interests. The authors declare no conflict of interests. 


\section{References}

1. International Agency for Research on Cancer. GLOBOCAN 2018. URL: https://www.iarc.fr/.

2. Franceschini G., Sanchez A.M., Di Leone A., Magno S., Moschella F., Accetta C., Natale M., Di Giorgio D., Scaldaferri A., D'Archi S., Scardina L., Masetti R. Update on the surgical management of breast cancer. Ann Ital Chir 2015; 86(2): 89-99.

3. Driul L., Bernardi S., Bertozzi S., Schiavon M., Londero A.P., Petri R. New surgical trends in breast cancer treatment: conservative interventions and oncoplastic breast surgery. Minerva Ginecol 2013; 65(3): 289-296.

4. Franceschini G., Martin Sanchez A., Di Leone A., Magno S., Moschella F., Accetta C., Masetti R. New trends in breast cancer surgery: a therapeutic approach increasingly efficacy and respectful of the patient. G Chir 2015; 36(4): 145152, https://doi.org/10.11138/gchir/2015.36.4.145.

5. Alfano R.R. Advances in optical biopsy for cancer diagnosis. Technol Cancer Res Treat 2011; 10(2): 101, https:// doi.org/10.7785/tcrt.2012.500184.

6. Nie Z., An R., Hayward J.E., Farrell T.J., Fang Q. Hyperspectral fluorescence lifetime imaging for optical biopsy. J Biomed Opt 2013; 18(9): 096001, https://doi.org/10.1117/1. jbo.18.9.096001.

7. Boppart S.A., Richards-Kortum R. Point-of-care and point-of-procedure optical imaging technologies for primary care and global health. Sci Transl Med 2014; 6(253): 253rv2, https://doi.org/10.1126/scitransImed.3009725.

8. Li G., Li H., Duan X., Zhou Q., Zhou J., Oldham K.R., Wang T.D. Visualizing epithelial expression in vertical and horizontal planes with dual axes confocal endomicroscope using compact distal scanner. IEEE Trans Med Imaging 2017; 36(7): 1482-1490, https://doi.org/10.1109/tmi.2017.2673022.

9. Gao Z., Li G., Li X., Zhou J., Duan X., Chen J., Joshi B.P., Kuick R., Khoury B., Thomas D.G., Fields T., Sabel M.S., Appelman H.D., Zhou Q., Li H., Kozloff K., Wang T.D. In vivo near-infrared imaging of ErbB2 expressing breast tumors with dual-axes confocal endomicroscopy using a targeted peptide. Sci Rep 2017; 7(1): 14404, https://doi. org/10.1038/s41598-017-13735-z.

10. Perry S.W., Burke R.M., Brown E.B. Two-photon and second harmonic microscopy in clinical and translational cancer research. Ann Biomed Eng 2012; 40(2): 277-291, https://doi.org/10.1007/s10439-012-0512-9.

11. König K., Riemann I., Ehlers A., Bückle R., Dimitrow E., Kaatz M., Fluhr J., Elsner P. In vivo multiphoton tomography of skin cancer. Proc. SPIE, Multiphoton Microscopy in the Biomedical Sciences 2006; 6089: 60890R, https://doi. org/10.1117/12.646000.

12. Jain M., Robinson B., Scherr D., Sterling J., Lee M., Wysock J., Rubin M., Maxfield F., Zipfel W., Webb W., Mukherjee S. Multiphoton microscopy in the evaluation of human bladder biopsies. Arch Pathol Lab Med 2012; 136(5): 517-526, https://doi.org/10.5858/arpa.2011-0147-oa.

13. Yan J., Zhuo S., Chen G., Milsom J.W., Zhang H., Lu J., Zhu W., Xie S., Chen J., Ying M. Real-time optical diagnosis for surgical margin in low rectal cancer using multiphoton microscopy. Surg Endosc 2014; 28: 36-41, https://doi. org/10.1007/s00464-013-3153-7.

14. Stanciu S.G., Xu S., Peng Q., Yan J., Stanciu G.A., Welsch R.E., So P.T., Csucs G., Yu H. Experimenting liver fibrosis diagnostic by two photon excitation microscopy and bag-of-features image classification. Sci Rep 2014; 4: 4636, https://doi.org/10.1038/srep04636.

15. Wu Y., Fu F., Lian Y., Chen J., Wang C., Nie Y., Zheng L., Zhuo S. Monitoring morphological alterations during invasive ductal breast carcinoma progression using multiphoton microscopy. Lasers Med Sci 2015; 30(3): 11091115, https://doi.org/10.1007/s10103-015-1712-y.

16. Denk W., Strickler J.H., Webb W.W. Two-photon laser scanning fluorescence microscopy. Science 1990; 248(4951): 73-76, https://doi.org/10.1126/science.2321027.

17. Cicchi R., Kapsokalyvas D., De Giorgi V., Maio V., Van Wiechen A., Massi D., Lotti T., Pavone F.S. Scoring of collagen organization in healthy and diseased human dermis by multiphoton microscopy. J Biophotonics 2010; 3(1-2): 34-43, https://doi.org/10.1002/jbio.200910062.

18. Cicchi R., Matthäus C., Meyer T., Lattermann A., Dietzek B., Brehm B.R., Popp J., Pavone F.S. Characterization of collagen and cholesterol deposition in atherosclerotic arterial tissue using nonlinear microscopy. J Biophoton 2014; 7(1-2): 135-143, https://doi.org/10.1002/jbio.201300055.

19. Gubarkova E.V., Kirillin M.Y., Dudenkova V.V., Timashev P.S., Kotova S.L., Kiseleva E.B., Timofeeva L.B., Belkova G.V., Solovieva A.B., Moiseev A.A., Gelikonov G.V., Fiks I.I., Feldchtein F.I., Gladkova N.D. Quantitative evaluation of atherosclerotic plaques using cross-polarization optical coherence tomography, nonlinear, and atomic force microscopy. J Biomed Opt 2016; 21(12): 126010, https://doi. org/10.1117/1.jbo.21.12.126010.

20. Wu S., Li H., Yang H., Zhang X., Li Z., Xu S. Quantitative analysis on collagen morphology in aging skin based on multiphoton microscopy. J Biomed Opt 2011; 16(4): 040502, https://doi.org/10.1117/1.3565439.

21. Osman O.S., Selway J.L., Harikumar P.E., Stocker C.J., Wargent E.T., Cawthorne M.A., Jassim S., Langlands K. A novel method to assess collagen architecture in skin. BMC Bioinformatics 2013; 14(1): 260, https://doi.org/10.1186/14712105-14-260.

22. Zipfel W.R., Williams R.M., Christie R., Nikitin A.Y., Hyman B.T., Webb W.W. Live tissue intrinsic emission microscopy using multiphoton-excited native fluorescence and second harmonic generation. Proc Natl Acad Sci U S A 2003; 100(12): 7075-7080, https://doi.org/10.1073/pnas.0832308100.

23. Malandrino A., Mak M., Kamm R.D. Moeendarbary E. Complex mechanics of the heterogeneous extracellular matrix in cancer. Extreme Mechanics Letters 2018; 21: 25-34, https:// doi.org/10.1016/j.eml.2018.02.003.

24. Luparello C. Aspects of collagen changes in breast cancer. Journal of Carcinogenesis \& Mutagenesis 2013; S13: 007, https://doi.org/10.4172/2157-2518.s13-007.

25. Mnikhovich M.V. Cell and cell-matrix interactions in breast carcinoma: the present state of problems. Rossiyskiy mediko-biologicheskiy vestnik im. akademika I.P. Pavlova 2014; 22(2): 152-161, https://doi.org/10.17816/ pavlovj20142152-161.

26. Clark A.G., Vignjevic D.M. Modes of cancer cell invasion and the role of the microenvironment Curr Opin Cell Biol 2015; 36: 13-22, https://doi.org/10.1016/j.ceb.2015.06.004.

27. lovino F., Ferraraccio F., Orditura M., Antoniol G., Morgillo F., Cascone T., Diadema M.R., Aurilio G., Santabarbara G., Ruggiero R., Belli C., Irlandese E., Fasano M., Ciardiello F., Procaccini E., Lo Schiavo F., Catalano G., De Vita F. Serum vascular endothelial growth factor (VEGF) levels correlate with tumor VEGF and 
p53 overexpression in endocrine positive primary breast cancer. Cancer Invest 2008; 26(3): 250-255, https://doi. org/10.1080/07357900701560612.

28. Han X., Burke R.M., Zettel M.L., Tang P., Brown E.B. Second harmonic properties of tumor collagen: determining the structural relationship between reactive stroma and healthy stroma. Opt Express 2008; 16(3): 1846-1859, https://doi. org/10.1364/oe.16.001846.

29. Aboussekhra A. Role of cancer-associated fibroblasts in breast cancer development and prognosis. Int $J$ Dev Biol 2011; 55(7-9): 841-849, https://doi.org/10.1387/ ijdb.113362aa.

30. Provenzano P.P., Inman D.R., Eliceiri K.W., Knittel J.G., Yan L., Rueden C.T., White J.G., Keely P.J. Collagen density promotes mammary tumor initiation and progression. BMC Med 2008; 6(1): 11, https://doi.org/10.1186/1741-7015-6-11.

31. Provenzano P., Eliceiri K.W., Yan L., Ada-Nguema A., Conklin M.W., Inman D.R., Keely P.J. Nonlinear optical imaging of cellular processes in breast cancer. Microsc Microanal 2008; 14(6): 532-548, https://doi.org/10.1017/ s1431927608080884.

32. Conklin M.W., Eickhoff J.C., Riching K.M., Pehlke C.A., Eliceiri K.W., Provenzano P.P., Friedl A., Keely P.J. Aligned collagen is a prognostic signature for survival in human breast carcinoma. Am J Pathol 2011; 178(3): 1221-1232, https://doi. org/10.1016/j.ajpath.2010.11.076.

33. Burke K., Tang P., Brown E. Second harmonic generation reveals matrix alterations during breast tumor progression. J Biomed Opt 2013; 18(3): 031106, https://doi. org/10.1117/1.jbo.18.3.031106.
34. Ambekar R., Lau T.Y., Walsh M., Bhargava R., Toussaint K.C. Jr. Quantifying collagen structure in breast biopsies using second-harmonic generation imaging. Biomed Opt Express 2012; 3(9): 2021-2035, https://doi.org/10.1364/ boe.3.002021.

35. Wu X., Chen G., Lu J., Zhu W., Qiu J., Chen J., Xie S., Zhuo S., Yan J. Label-free detection of breast masses using multiphoton microscopy. PLoS One 2013; 8(6): e65933, https:// doi.org/10.1371/journal.pone.0065933.

36. Natal R.A., Vassallo J., Paiva G.R., Pelegati V.B., Barbosa G.O., Mendonça G.R., Bondarik C., Derchain S.F., Carvalho H.F., Lima C.S., Cesar C.L., Sarian L.O. Collagen analysis by second-harmonic generation microscopy predicts outcome of luminal breast cancer. Tumor Biol 2018; 40(4): 1010428318770953, https://doi. org/10.1177/1010428318770953.

37. Wang Y., Lu S., Xiong J., Singh K., Hui Y., Zhao C., Brodsky A.S., Yang D., Jolly G., Ouseph M., Schorl C., DeLellis R.A., Resnick M.B. ColXa1 is a stromal component that colocalizes with elastin in the breast tumor extracellular matrix. J Pathol Clin Res 2019; 5(1): 40-52, https://doi. org/10.1002/cjp2.115.

38. Uchiyama S., Fukuda Y. Abnormal elastic fibers in elastosis of breast carcinoma. Ultrastructural and immunohistochemical studies. Acta Pathol Jpn 1989; 39(4): 245-253.

39. Elasbali A.M., Al-Onzi Z., Hamza A., Khalafalla E., Ahmed H.G. Morphological patterns of elastic and reticulum fibers in breast lesions. Health 2018; 10(12): 1625-1633, https://doi.org/10.4236/health.2018.1012122. 